\title{
A Surveyof human face detection methods
}

\author{
Farah Qais Abdulalla Asmaa Sadiq abduljabar \\ Department of computer \\ college of science \\ University of al-mustansryia \\ Baghdad,Iraq
}

\author{
Shaimaa Hameed Shaker \\ Department of computer \\ science \\ University of Technology \\ Baghdad,Iraq
}

Recived : 17\4\2018

Revised : $26 \backslash 4 \backslash 2018$

Accepted : $3 \backslash 5 \backslash 2018$

Available online : 3/6/2018

DOI: $10.29304 / j q c m .2018 .10 .2 .392$

\begin{abstract}
The aim of this research is to produce a critical survey on detecting and extracting human face with the features over the past 10-15 years. Face Detection is one of the most common techniques in various future visual applications, such as teleconferencing, facial recognition systems, biometrics and human computer interface, not only because of the difficult nature of the face as an object, but also because of the myriad applications that require face detection application as a first step .

Finally, we offer a facial detection technology based on skin color segmentation as well as the facial features inside it. The determination of the human face as elliptical area was achieved. Meanwhile, several techniques were used such as enhancement, thresholding, edge detections and binarization techniques to achieve the aim of the suggested method. The facial features are detected and extracted inside the elliptical area; these features can be categorized in three parts: Nose, Mouth and lips localization. The features are detected and extracted in the human face based on image processing techniques.
\end{abstract}

Keywords: Face Detection, Facial Features, Image Based Methods, Feature Based Method, Image Processing 


\section{Introduction}

One of the most concerted biometric techniques is a face detection, which can be used in many applications such as Identification of persons, access to government institutions, measurement of breathing and surveillance [1]. Human facial extraction techniques are the most important and decisive step within the image processing, artificial intelligent and pattern recognition. The concepts of these techniques are looking for significant information in the human image [2].

This paper provides a review of the human face detection methods as well as the facial features. The area of the face and features detection become a common in the fields of the research due to their importance and multiple applications. Facial detection and extraction techniques suffer from many limitations that may be have a clear impact on these techniques, such as skin color, facial hair, wearing glasses and facial expressions. Additionally light conditions and the head movements in an image are the most common and difficult problems [3]. The human face is changeable and cannot be easily detected or recognized, this problem complicates computer work with the help of the recognition or detection system [4]. So many researchers have been looking into this area to find advanced ways to develop this field [5]. The methods proposed for human face detection can be categorized into two fields: Feature-based methods and Image based methods. Where Feature-based methods are classified in three groups: Active shape model, low level analysis and feature analysis methods while image based methods are classified in three groups: Neural network, Linear Sub Space and Statistical Approach methods [6].

Table 1: Illustrated Various Face Detection methods

\begin{tabular}{|c|c|c|}
\hline \multirow{4}{*}{$\begin{array}{c}\text { 1-Feature } \\
\text { Base Methods }\end{array}$} & Active & Snakes \\
\cline { 3 - 3 } & shape Model & Templates \\
\cline { 3 - 3 } & & PDM \\
\cline { 3 - 3 } & $\begin{array}{c}\text { Low level } \\
\text { analysis }\end{array}$ & Skin color \\
\cline { 3 - 3 } & Neural Network & Edge \\
\hline \multirow{4}{*}{$\begin{array}{c}\text { 2-Image } \\
\text { Based } \\
\text { Methods }\end{array}$} & Linear subspace & Eigen Face \\
\cline { 2 - 3 } & $\begin{array}{c}\text { Statistical } \\
\text { Approach }\end{array}$ & PCA \\
\cline { 2 - 3 } & & SVM \\
\cline { 2 - 3 } & &
\end{tabular}

\section{FEATURE BASE METHODS}

Recognize the objects based on their features. Detect and recognize the human face from the other things based on the human features such as eyes, mouth and nose, and then used this features to detect human face. The statistical classifiers are helps to distinguish between the face and nonface. Although there are many method has been suggested to extract the facial features which illustrated in the literature, most of them faced the limitations such as the lights, low resolution occlusion and noise.

Most research and studies have shown that skin color is an important element of facial detection among other objects although different people have different color skin and it is more cleared when people race is also a measure of evaluation [7].

\subsection{Active Shape Model}

\subsubsection{Snakes}

Snakes or Active contours are usually implemented to locate the boundary of the head, and then the features related to the located boundary can be established by these contours. This can be accomplished by placing the starting point of the snake at the proximity around the head's borders, after the snake stands on the nearby edges and then assumes the shape of the face. F snake is defined as:

$$
\mathrm{E}_{\text {snake }}=\mathrm{E}_{\text {internal }}+\mathrm{E}_{\text {external }}
$$

The $\mathrm{F}$ internal is the internal energy that presents the part that depends on the intrinsic properties of the snake and defines its natural evolution, while the external energy $\mathrm{F}$ external responds the internal energy and allows the contours to deviate from the natural evolution and eventually assume the shape of nearby features. Although this method is perfect in detecting human face, it may be faced two limitations such as: a snake doesn't work very well in recognize non convex features because their tendency to link minimum curvature. Also, sometimes part of contour gives incorrect features for some images [8],[4] 


\subsubsection{Deformable template-based approach}

One of the major reasons that makes computer aided detection or recognition system complicated, is the difficulty in recognizing the variable structures in human face [4]. One simple solution to this problem would be based on matching the test image with a template. This approach was modified by [9], in this approach, the authors used the template-based approach to locate the human face based on selecting four features (whole face, nose, mouth and eyes) as template per person.

The recognition technique was achieved by comparing the test image with the database images. Correlation was computed for each feature template; a vector of matching scores for each feature was calculated. The test subject was then classified as the subject with the highest score. This approach introduced a significant method to store the face area by discrimination ability (eyes, nose and mouth in order). This method succeeded in recognizing a number of people, it had some limitations because it deals only with the static and frontal images, so it was unable to deal with head movements. Additionally the illumination must be controlled; the same powerful light was used for the test and data base images.

\subsubsection{PDM (Point Distributed Models)}

The flexible model (PDMs) was created by [10]. It is quite different from the other models in the application process. The PDMs are compact parameterized descriptions of a shape based upon statistics. The bounding shape of PDM is categorized into a group of labeled points, and then the differences of these points can be described over a training set that contains objects of various sizes and poses.

One of the proposed methods was suggested by

Wiscott et al. [45], they based on a well-known feature as a method of matching the diagram to a flexible package proposed. The difference in lighting and contrast played important role in success the "Gabor filters" to remove the noise and the changeability in the image. A graph is generated for each face separately as follows: on the face, a group of fiducially points were chooses. Each fiducially point is a node of a completely connected diagram, called with "Gabor filters" then apply a window around the fiducially points.
This technology has really enhanced facial recognition performance under variations of pose, angle, and expression.

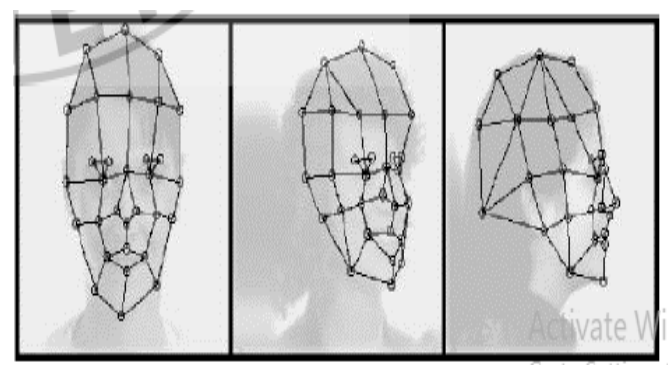

Figure1: Grids for face recognition[46]

The face PDM was applied to present the global appearance of the face with the facial features such as (nose, eyes, mouth and eyebrows ). Moreover it can be used to detect multiple faces and has several implementations such as coding and facial expression interpretation [4],[11]. On other hand the detection of face features automatically is considered as one of the difficulties in this approach.

\subsection{Low level Analysis}

\subsubsection{Skin -color method}

Skin color segmentation can be considered as an important feature to detect human face as well as feature extraction. For this purpose, It can be considered as a rapid technique than others. The color is continual orientation under specific lighting conditions. This effects makes motion valuation much easier, the reason was only the translation model is needed to estimate the motion [12]

Skin color can be considered as a significant feature that can be used to detect human face. It has proved effective because it gives a fast detection and provides robust results enough to perform real-time tracking [13]. This process depends on the color of the human face which can be tracked and distinguished from the color of other objects in the scene and the color of the background. 
[14] proposed a new approach to detect human face as well as facial feature, the two major steps that has been proposed to detect the human face in the suggested images are : Initially, the skin region was segmented from the image using RGB, YCbCr, CEILAB (L*a*b) and HSV color models. The skin region was tested using color models and the knowledge of geometrical properties of human face while the non-skin region was removed using color models with thresholds. The test results showed the distinctive effect of this algorithm in detecting human face.

The skin color segmentation was used from many researchers to detect and recognize the human face from the color images such as [15][16]-[17]-[18]-[19]

However significant limitations of this method appeared when there is a wide variety of skin colors, light conditions and there are problems with grey level images. Sometimes it can be used as a first step in face detection, but is not suitable for high level feature extraction and analysis.

\subsubsection{Motion base}

When using the video sequences, the moving object can be detected based on the motion information [20]. The simply thresholding accumulated frame can be used to extract the Animated silhouettes such as body parts and faces. The facial features can be located and extracted by frame differences besides face regions [21].

\subsubsection{Edge}

A Laplacian edge detection operator was performed to detect the facial features (nose, eyes, mouth and chin contour) from gray level images [22]. In [23] introduced an algorithm to detect further head outline in addition to the locations of facial features that includes eyes, eyebrows and mouth from a grey-scale image where the edge detectors were used to obtain the face curve. [24] proposed a novel method to detect human face in thermal image as well as the tip of the nose.

The aim of this project was extracted human face from the background and then determined the area under the tip of the nose after detect the nose region. This area was used to measure the respiration rate because it is more affected than other regions by breathing process
Initially, the human face was enhanced by using average-low-passfilter to reduce the noise from the image. The prewitt operator was used to segment the face boundaries from the background. The prewitt operator was used to extract the boundaries in horizontal and vertical directions of the human face. After extract the boundaries of the human face, the nose region was extracted based on the eye regions as well as the temperature of this area. Then the area (region of interest ROI) under the tip of the nose was detected and extracted as circle regions. Then the respiration signal was extracted by averaging the pixel values within the ROI for each image and the resulting information was plotted against time.

In general the facial features such as eyebrows and lips seem darker than surrounding regions in the face, then this techniques can be implemented for local minima, meanwhile the local maxima has been implemented to marker the bright facial spots like nose tips then the gray-scale thresholding can be performed for final detection [23]-[25]- [26].

\section{IMAGE- BASE METHODS}

\subsection{Neural network}

[27] proposed another suggestion method for detecting human face using a neural network.

This method was suggested to deal with the light problem in the face detection process. The image was segmented into several regions. The neural network was also used to classify the regions into two groups, either face or no face. Pre-processing steps were applied for each region by using a light correction algorithm and histogram equalization techniques. The light correction needed a statistical analysis for the background color to be approximately estimated across the image. While the histogram equalization techniques were used to adjust the histogram of the image in a variety of ways either by averaging the histogram for an image or enhancing the contrast of image. The light condition and skin color problems remained problems with the face detection technique.

The development of this method was suggested by [28]. The facial expressions was detected and located in the human face. All the extracted features from the human face were inputted to Neural-Network to detect the decision of being Neutral face or non- Neutral. 
Facial expression analysis automatically combines a number of tasks which includes face detection, facial features extraction and facial features represented. Based on this analysis the face was classified as Neutral face or NonNeutral. After the face region was extracted by using skin-color technique. Detection and extraction were focused on the six facial point features from the image which were less affected by noise than other parts of the images. The features includes: -two pupil centers, two eyebrow inner end-points and the two corners of the mouth. The distance between these points was calculated. Further, the features of the eyes and the mouth were extracted by an ellipse fitting algorithm and the histogram technique respectively. All the features were inputted to Neural-Network to detect the decision of being Neutral or non- Neutral. This approach was an important technique to reduce the error rates for face recognition, because universal expressions are one of the important reasons for inaccuracy in the recognition process. This approach could detect the face and extract the facial expression and recognition.

\subsection{Eigen faces method as a Linear Sub Space}

[29] was present an early example of employing eigen-vectors in face detection and face recognition. The recognition based on a simple neural network for face images. The basis of the eigenfaces method is the Principal Component Analysis (PCA). Eigen-faces and PCA have been used to represent the face images efficiently [30].

[31] suggested using eigenface technique to detect and recognize the human face from the color image. This technique was based on calculation of the Euclidian distances between the new eigenface and the previous one. The smallest Euclidean distance is person resembles the most. Although the results showed that sometimes failure occurs, but it have success rate about of $94.7 \%$ when applied it to the large database.

Two approaches were suggested from [3] to detect the position of the human face area using the genetic algorithm and detecting the facial features using the eigenface technique. The authors tried to solve several problems such as the orientation of the face, skin color and the lighting effect. The eye regions play an important role on detecting the facial region and facial features based on the characteristics of eye regions
Genetic algorithm and the eigenface techniques were also applied. The possible face regions were determined by using the genetic algorithm, whilst the fitness of these regions was determined using eigenface. . To reduce the searching space, the human eye regions were selected by testing all the valley regions in an image using a genetic algorithm. The pair of possible eye candidates was used to segment the face regions.

The size of human face $\left(Z_{\text {face }}\right)$ is proportional to the distance between two eyes $\left(E_{\text {eye }}\right)$ as in Equation (2)

$$
Z_{\text {face }}=1.8 E_{\text {eye }}
$$

The relationship between the facial features (the eyebrows, eyes, nose, and mouth) play significant role in determined the possible face region.. The facial features are then extracted from the detected face regions. The eigenfaces was used to calculate the fitness value for each candidate face. A suitable candidate region was chosen after a number of repetitions, and then features were extracted from this region. The selected face was then further verified by measuring the symmetries and determining the existence of the different facial features.

The significant progress in the performance of this method was proposed by [32]. He suggested the algorithm to detect human face by calculate the eigenvalues and eigenvectors of the face images. This calculation depended on locating the human face as well as the eyes in the image uses Principal Component Analysis. This method was succeeded in detecting human faces, and feature extractions in simple and complicated background.

Most researchers have used eigenface and eigenvector to locate the human face and facial features from the color images such as [34]-[35][36]-[37].

\subsection{Statistical Approach}

\subsubsection{Principle Component Analysis (PCA)}

PCA technique was introduced firstly by [38] which based on the Eigen- faces. [38]. [36] suggested using PCA technique to detect and recognize human face. In this approach the eigenface was extracted for each image in the training set by represented each image in the training set as a linear combination of weighted eigenvectors. 
These eigenvectors are obtained from covariance matrix of a training image set. Weights are found after selecting the most important set of faces. The classification is done by measuring minimum Euclidean distance that was achieved by projected each test image onto the subspace spanned by the eigen-faces to perform the recognition and detection process [39]. This technique has the advantage to keep the important information of image and rejects the redundant information therefore it considers as an important technique in extraction the facial features from the images. The PCA was used to reduce the large dimensionality of observed variable to the smaller fundamental dimensionality of independent variable without losing much information. This technique was widely used from many researchers such as [40],[41].

\subsubsection{Support Vector Machine (SVM)}

This technique is considered as a linear classifier, in the last 50 years the SVMs become a widely applied classification technique. It played an important role in object detection, and recognition [15]. This technique was work by firstly scanned the images to detect the faces, then scaled the faces image into the image's patches. The filtered image (output image) is used as input to the recognition classifier which the code expression into different dimension. The SVM trained after selected the facial features [42]. [1] proposed a new technique to detect and recognize human face by using Support vector machines (SVM). They tried to solve the problem of face detection and face recognition by using SVM with binary tree. They tried to compare SVM with other techniques; the experimental results showed that the SVMs are a better learning algorithm than the nearest center Classification approach (NCC) criterion for face recognition.

A modified method to detect the human face in visible domain using the skin colour was proposed by [42]. They also used a skin colour to detect the human face region. They suggested another technique to reduce the effect of significant limitations of the skin colour by using a high-pass filter. This filter of the wavelet transforms was used to highlight the edges of the selected areas.

Vertical, Horizontal, and filter-like projections were represented as feature vectors. The element vectors were then categorized as face or non-face using either vector support techniques or dynamic programming. Dynamic programming is a classification technique that is used to calculate the best possible path matching between the original feature vector and template feature vector.

The Euclidean distance was used to measure the similarity between these two vectors. The decision is made as face or no-face depends on thresholding the resulting distance.

In this approach the authors attempted to demonstrate that the use of vector machine technique is more appropriate than dynamic programming in face detection process as it is more accurate, faster and cheaper.

[43] tried to achieve sufficient speed and accuracy in SVM techniques; they tried to build a component-based face detector in online face recognition system using SVM classifiers.

Finally, a method was suggested to detect human face as well as feature extraction, human face was detected based on skin color segmentation and then extracted as ellipse region. The nose region was located and extracted as rectangular region in the elliptical facial area. This technique was achieved by threshold and binarization the extracted area and then looking for the longest vertical line.

As the Mouth region represented the bottom part of the face therefore, it was detected based on thresholding the elliptical area, then the mouth area was located by searching the area under the tip of the nose. The longest horizontal line represents the center line of the lips. This area was extracted as rectangular area. The lips were detected in the mouth region based on the threshold techniques as well as the points of the lips corners [44]. Fig.2 shows the extraction of the human face as an elliptical region, the nose as rectangular region while the mouth as an elliptical region with lips. 

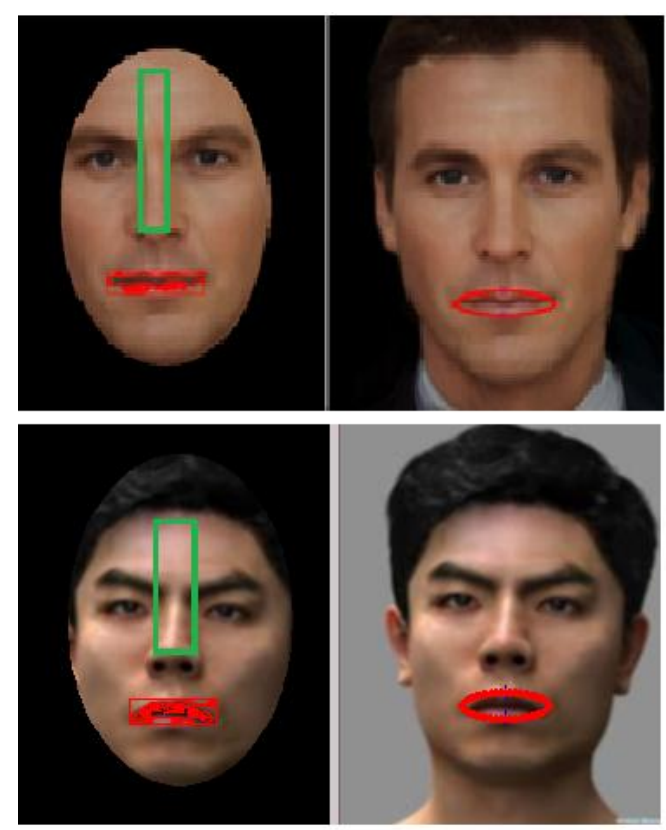

Figure2: Detecting The Human Face As Elliptical Area As Well As The Mouth And The Nose Regions In The Human Face

\section{Conclusions}

Detecting facial features within human face is an important task when dealing with image processing techniques. Facial detection methods can be sorted through a number of methods depending on how they are used and operated.

In this paper, they are classified into featurebased methods and image-based methods. The paper has introduced the advances has made to improve the performance of face detection with facial features.

Finally, we suggested a method to detect and extract human face as elliptical area from the background. The facial features within the face area were located and extracted sequentially. Image processing such as (enhancement, binarazation, thresholding segmentation and edge detection) played an important role to achieve the aims of our proposal.

\section{References :}

[1] Guo G., Li Z. and Chan K.,"Face Recognition by Support Vector Machines". Proceeding Fourth IEEE International Conference on Automatic Face and Gesture Recognition, p.p. 196-201.

[2] Nixon S. M. and Aguado S. A., 'Feature Extraction and Image Processing", Handbook 1st Edition, Academic Press is an imprint of Elsevier. 2002.

[3] Wong W. K., Lam M. K. and Siu C. W.,"An Efficient Algorithm for Human Face Detection and Facial Feature Extraction under Different Condition.", Pattern Recognition, Department of Electrical and information, the Hong Kong University. Pattern Recognition, Vol. 34, No. 10. 2004.

[4] Hjelmas E., Low K. B.,"Face detection: A survey". Computer Vision and Image Understanding, Vol. 83, No. 3, 2004,p.p.236274.

[5] Pan H., Zhu Y. and Xia L., "Efficient and accurate face detection using heterogeneous feature descriptors and feature selection", Computer Vision and Image Understanding, vol. 117(1), 2013, p.p. 12-28.

[6] Yousra B. JEMAA; Sana K. "Automatic local Gabor features extraction for face recognition", (IJCSIS) International Journal of Computer Science and Information Security, Vol. 3, No. 1, 2009.

[7] Li Z., Xue L. and Tan F., "Face detection in complex background based on skin color features and improved AdaBoost algorithms", in Informatics and Computing (PIC), 2010 IEEE International Conference on, vol. 2, December 2010, p.p. 723-727.

[8] Modil, M., \& Macwan, F."Face detection approaches: A survey. International journal of innovative research in science, engineering and technology",2014.

[9] Brunelli R. and Poggio T., "Face Recognition: Features Versus Templates". Pattern Analysis and Machine Intelligence, IEEE Transactions. Vol. 15, No. 10,1993, p.p. $1042-1052$.

[10] Lanitis A. , Taylor C.J and Cootes T.F.,' Automatic tracking, coding and reconstruction of human faces, using flexible appearance models', Institution of Engineering and Technology, DOI: 10.1049/el:19941110, Vo. 30, No.19, 15 Sep 1994. 
[11] Rath, S. K., and Rautaray, S. S.,"A Survey on Face Detection and Recognition Techniques in Different Application Domain", International Journal of Modern Education and Computer Science, Vo.6, No. 8, 2014.

[12] Sanjay K. ,Singh D., Chauhan S., MayankV., Richa S. ,'A Robust, "Skin Color Based Face Detection Algorithm", Tamkang Journal of Science and Engineering, Vol. 6, No. 4, 2003,pp. 227-234.

[13] Bovik C.A. , "The Essential Guide to Image Processing. Handbook Communications", Networking, and Multimedia. 2nd Edition, Elsevier Inc USA, 2009.

[14] Devendra S. R., Dheeraj A., Human Face Detection by using Skin Color Segmentation, "Face Features and Regions Properties", International Journal of Computer Applications . Vo. 38, No. 9, January 2012.

[15] Sathya V. and Chakravarthy T., "A Survey on Various Methods of Face Detection Feature Extraction and Classification for Facial Expression Recognition", International Journal of Innovative Research in Computer and Communication Engineering Vol. 4, No 5, October 2016.

[16] Arti K., Sowmya R. and Swetha R., "Face Detection Using Color Based Segmentation and Morphological Processing: A Case Study," Int. Symposium on Computer Egg. \& Tech., 20 March, 2010.

[17] Dilip D., Yogesh T., Pramod K. and D.V.B Singh, "'Human Face Detection By YCbCr Technique", International Journal of Emerging Technologies in Computational and Applied Sciences (IJETCAS), 2013.

[18] Prashanth K. G, , Shashidhara M., "Skin color segmentation for detecting human face region in image", International Conference on Communication and Signal Processing, 2014 , pp. $001-005$.

[19] Rosali M. and Raghunadh M.V , "Skin Color Segmentation based Face Detection using Multi-Color Space", international Journal of Advanced Research in Computer and Communication Engineering, Vol. 5, No 5, May 2016.

[20] Low B. K. , Ibrahim M. K, "A Fast and Accurate Algorithm Facial Feature Segmentation", 1997.
[21] Sunita R., Sudipta R. and Bandyioahyay S., "A tutorial Review on face Detection", International journal engineering research \& technology (IJERT), Vol. 1, No. 8, 2012.

[22] Sakai T., Nagao M., and Kanade T., "Computer analysis and classification of photographs of human faces", in Proc. First USA-Japan Computer Conference, 1972, p.p. 2.7.

[23] Craw, I., Ellis, H. and Lishman, "Automatic extraction of face feature". Pattern Recog. Lett. 1831871987.

[24] AL-khalidi F. Q., Saatchi R., Burke D. and Elphick H.'Tracking human face features in thermal images for respiration monitoring", In: 2010 IEEE/ACS International Conference on Computer Systems and Applications (AICCSA), Hammamet, Tunisia, 16-19 May, 2010.

[25] Toshiyuki S., M. Nagao, Takeo K., "Computer analysis and classification of photographs of human face," First USA Japan Computer Conference, 1972.

[26] Bakshi, U., and Singhal, R., "A survey on face detection methods and feature extraction techniques of face recognition". International Journal of Emerging Trends \& Technology in Computer Science (IJETTCS), Vo. 3, No. 3,2014, p.p. 233-237.

[27] Rowley H., Baluja V., Kanade T., "Neural Network-based face detection". IEEE: Pattern Recognition, and Machine intelligence Proceedings. Vol. 20, No. 1, p.p., 1998, 23 38.

[28] Tian Y. and Bolle M.R. (2008) "Automatic Detection Neutral Face for Face Authentication and Facial Expression Analysis". Exploratory Computer Vision Group, In AAAI-03 Spring Symposium on Intelligent Multimedia Knowledge Management. [Online] last accessed on 2nd of April 2008 at: http://www.research.ibm.com/peoplevision/ $\mathrm{N}$ eutralface.pdf.

[29] Kohonen T., Self-Organization and Associative Memory. Springer 1989.

[30] Sirovich L. and Kirby M., "LowDimensional Procedure for the Characterization of Human Faces", Journal of the Optical Society of America, A 4 (1987) 519-524. 12. 
[31] Çarıkçı M. and , Özen F., "A Face Recognition System Based on Eigenfaces Method ", Procedia Technology , 2012, pp. $118-123$.

[32] Nupoor M. , 'Face Detection and Recognition using Eigen Faces by using PCA “ Int.J.Computer Technology \& Applications, Vol 4, No. 1, 2013,.p.p. 126129.

[33] Abd Rahman A., Noah M. A., Safar R. S. , Kamarudin N., "Human Face Recognition: An Eigenfaces Approach" International Conference on Advances in Intelligent Systems in Bioinformatics, ChemInformatics, Business Intelligence, Social Media and Cybernetics (IntelSys).Published by Atlantis Press.2014, p.p.42-47.

[34] Mittal N. \& Walia F., "Face recognition using improved fast PCA", IEEE Congress on Image and Signal Processing,Vol.1, 2008, pp. 554-558.

[35] Hossein A.S. , Heidari B.Z. \& Dehghani C.H., "A new face recognition using PCA, Lda and neural network", International Journal of Computer Science and Engineering, Vol. 2, No. 4,2008.

[36] Chandra L. P. , Al Sumam A., "Face Recognition Using Principal Component Analysis Method", International Journal of Advanced Research in Computer Engineering \& Technology (IJARCET) Vol.1, No. 9) November ,2012 p.p.2278 - 1323.

[37] Shemi P. M, Ali M., “A Principal Component Analysis Method for Recognition of Human Faces: Eigenfaces Approach", International Journal of Electronics Communication and Computer Technology (IJECCT),Vo. 2, No.3, 2012.

[38] Kirby M. and Sirovich L., "Application of the 'Karhunen-Loe've Procedure for the Characterization of Human Faces", IEEE Trans. Pattern Analysis and Machine Intelligence, vol. 12, No.1, Jan. 1990,pp. 103108.
[39] Liton C., Abdulla A, "Face Recognition Using Principal Component Analysis Method", International Journal of Advanced Research in Computer Engineering \& Technology (IJARCET) Vo.1, No. 9, 2012 .

[40] Chih-Chung Chang, Chih-Wei Hsu, and Chih-Jen Lin. "The analysis of decomposition methods for support vector machines". IEEE Transactions on Neural Networks, Vo.1, No.4,2000,p.p.10031008.

[41] Anand, B., " Face Recognition using SURF Features and SVM Classifier ", International Journal of Electronics Engineering Research. ISSN 0975-6450 Vo. 8, No. 1,2016,p.p. 1-8.

[42] Turkan M., Dulek B., Omaran I. and Cetin E, "Human Face Detection in Video Using Edge Projections", in Proceeding of the SPIE -the international society for optical Engineering: Visual Information Processing XV, Vo.. 6246, No.1., 2006.

[43] Kukenys I. , McCane B. , "Support Vector Machines for Human Face Detection", April, Christchurch, New Zealand NZCSRSC 2008.

[44] Al-khalidi F., "Facial Features Extraction From the Human Face", International Journal of Electronics Communication and Computer Engineering Vo.7, No.6, 2016,p.p. 2249071X.

[45] Wiskott L. , Fellous J.-M, Krüger N., and von der Malsburg C., "Face Recognition by Elastic Bunch Graph Matching," IEEE Transactions on Pattern Analysis and Machine Intelligence, Vol.19, pp.775-779, 1997.

[46] Brunelli R. , Poggio T. , ' Face recognition: features versus templates' , IEEE Transactions on Pattern Analysis and Machine Intelligence, Vol. 15, No. 10, October (1993), pp. 1042-1052. 


\section{مسح لطرق تحليد وجه الإنسان}

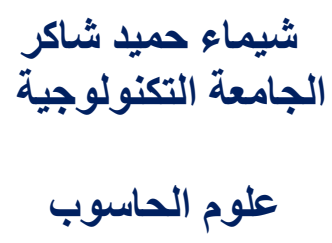

\author{
فرح قيس عبدالله اسماء صادق عبد الجبار \\ الجامعة المستنصرية \\ كلية العلوم \\ قسم الحاسبات
}

: (المستخلص : n

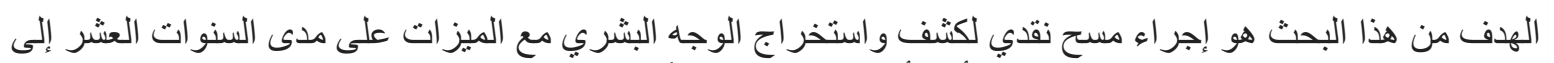

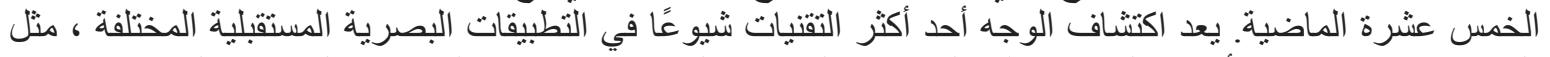

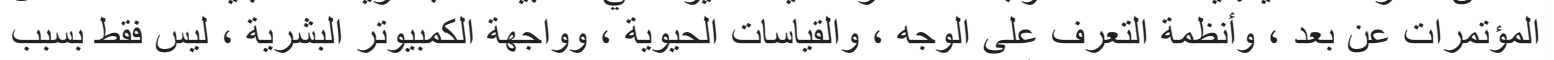

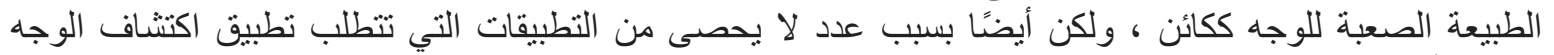
كخطوة أولى. و أخيرًا ، نقدم تقنية الكثف عن الوجه استنادًا إلى تجزئة لون البشرة بالإضافة إلى ميز ات الوجه الموجودة داخلها. تم

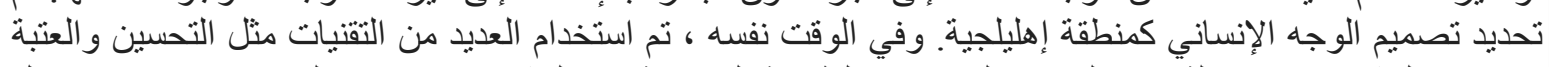

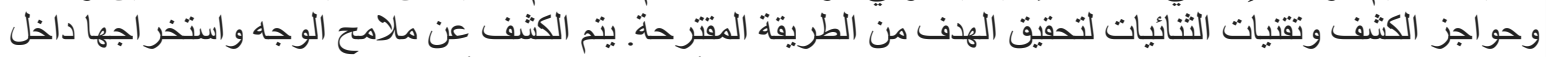

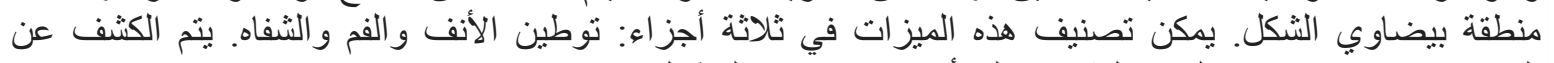

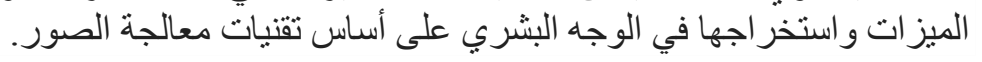

